\title{
Valuation of R\&D compound option using Markov chain approach
}

\author{
Guglielmo D'Amico ${ }^{1}$ (I) $\cdot$ Giovanni Villani ${ }^{2}$
}

Received: 17 March 2021 / Accepted: 18 May 2021 / Published online: 31 May 2021

(c) The Author(s) 2021

\begin{abstract}
Incorporation of technical risk in compound real options has been considered in Cassimon et al. (2011) concerning the valuation of multi-stage pharmaceutical R\&D. There, the technical success probabilities at each development stage were assumed to be generated independently of each other. This assumption can be unrealistic in many applied problems, pharmaceutical R\&D included. We present a valuation procedure dealing with dependent success probabilities and random development stage times. This greater flexibility allows a better description of the sequence of decision stages and results, which in turn, impact the value of the considered project. The theoretical results are illustrated through a numerical example that shows the implementation of the model to a pharmaceutical R\&D problem.
\end{abstract}

Keywords Real option · Technical risk - Compound option

JEL Classifications C02 - G11

\section{Introduction}

R\&D investments play a fundamental driving force to guarantee firms the innovations that allow them to adapt and survive in a rapidly changing economic environment. High-tech products, new drugs as vaccines, innovative production systems, require a high level of R\&D investments. The traditional approach to valuing such investments is the NPV (Net Present Value) criterion: if the project's NPV exceeds the investment

Guglielmo D'Amico

guglielmo.damico@unich.it

Giovanni Villani

giovanni.villani@uniba.it

1 Department of Economics, University of Chieti-Pescara, Viale Pindaro 42, 65127 Pescara, Italy

2 Department of Economics and Finance, University of Bari, Largo Abbazia S. Scolastica 53, 70124

Bari, Italy 
costs, one should invest; otherwise, if a project's NPV is not positive, the project should be rejected. However, traditional methods cannot properly grasp the managerial flexibility value that increases with the increase of uncertainty (see for example the studies by Newton et al. 2004 and Hartmann and Hassan 2006). A long period elapses from the starting of the original study to the obtention of the final products, with technical and competitive uncertainties spread far and wide. Most successful firms show that the flexibility to postpone a project, abandon or develop it based on market revealed information is a key factor that needs to be evaluated (see Myers 1984). In this context, the Real Options Analysis (ROA) meets the criteria required to support managers decisions offering an alternative that allows for incorporating the strategic issues and the profitable growth opportunities. ${ }^{1}$ Unlike most other types of investment problems, the uncertainty of $R \& D$ investments does not only come from economic circumstances as the discovery process is also highly uncertain. The two types of uncertainty are usually referred to as economic uncertainty and technological one.

In the academic literature, a number of papers have appeared dealing with these two types of uncertainty, among which it is worth to mention the one by Dixit (1988) and Weeds (2002). Dixit (1988) derives analytical expressions in order to value R\&D project in a context of race between heterogeneous firms by assuming that the hazard rate, the instantaneous probability of discovery, is constant over time. Weeds (2002) identifies a real option trade-off in a stopping game in which two players choose when to make an irreversible investment decision.

Although there is sufficient evidence showing management's awareness of the options thinking in the evaluation and selection of R\&D projects, researchers have been argued which theoretical real options models are more appropriate in valuing R\&D projects. Whenever the investment is made in a phased manner, with the commencement of the subsequent phase being dependent on the successful completion of the preceding phase, it is known as sequential investment. Each stage provides information for the next, thus creating an opportunity (option) for subsequent investment in a new technological area. Therefore, models have tended to treat R\&D investment as a sequential compound option and to value them using the techniques of 'Compound Options', also known as 'Option on Options'. In the case of a 2-fold, Geske (1979) computes a solution in continuous time of a call compound option. Carr (1988) proposes, in the extensions of his model, an $n$-fold compound exchange option that collapses into a traditional $n$-fold compound option assuming that the volatility of the delivery asset is equal to zero.

Following this research, Cassimon et al. (2004) propose a continuous-time real option analysis valuing a multi-staged pharmaceutical $R \& D$ which can be seen as a chain of

\footnotetext{
1 A critical aspect in the real option pricing approach is given by the impossibility to construct a replicating portfolio, as the assets are non tradable. In general, investment problems are much too complex to be modeled as a standard option, hence the option model must be tailor-made, with standard assumptions no longer applicable. Classic ROA is based on the assumption that the project can be replicated by a portfolio of market-driven instruments that are all exactly equivalent (Brennan and Schwartz 1985; Amram and Kulatilaka 1999). To solve this shortcoming, one issue can be to link the evaluation of a real project with quoted assets that have the same level of risk (see Borison 2005, Smith and Nau 1995). In fact, many manager intuitively feel that NPV-based valuation has flaws when handling multi-stage R\&D projects and therefore even negative NPV project are undertaken, since it does not consider the value of opportunities to wait and revise investment strategy (see e.g. Trigeorgis 1993).
} 
real options. Their model offers a closed-form solution for the valuation of new drug development using a generalized $n$-fold compound option model, but implicitly bundle both commercial and technical risk in one risk measure. Besides, Cassimon et al. (2011) extend their previous model, by explicitly incorporating technical risk. The closedform formula is still preserved for an $n$-fold compound option. In both papers, the technical success probabilities at each development stage are assumed to be generated independently from each other. Kelloss and Charnes (2000) explain the decision-tree method and binomial lattice method and use them to value a biotechnology company as the sum of the values of its drug-development project. However, Kelloss and Charnes (2000) consider the technical uncertainty to be deterministic to value the growth option of a drug project.

Our paper aims to propose a valuation procedure dealing with dependent success probabilities and random development stage times. This greater flexibility allows a better description of the sequence of decision stages and results that in turn impact the value of the considered project. Our methodology is based on Markov chains in continuous time, which allows the management to receive a more general and efficient description of the technical risk process. This choice is motivated by the high versatility demonstrated by Markov chains and some of its generalizations in describing different real life problems, see e.g. Ortobelli Lozza et al. (2011), Barbu and Karagrigoriou (2018), D’Amico et al. (2019) and De Blasis (2020).

To the best of our knowledge, the combination of real options and the Markov chains model represents an innovative approach that has few precedents. For instance, Elliott et al. (2009) investigate the investment timing problem with technical uncertainty considering project costs via Markov chains. Song et al. (2017) employ an irreversible regime-switching Markov chain to model the multi-stage and technology life cycle of the project in the high-tech industry. Maier et al. (2020) study a large portfolio of options (deferment, staging, mothballing, abandonment) under conditions of four underlying uncertainties. They model as stochastic Markovian processes, the evolution of endogenous uncertainties depending on the decision maker's strategy or the system's state.

Thus, in summary, this work advances the knowledge of how technical risk impacts the value of a real option in a realistic scenario where success probabilities at each stage are generated according to a Markov process. The Markov process modulates the timeline of decision times. This allows us to reject usual limiting and simplifying assumptions of independence of success probabilities and deterministic timeline of action times which are, at authors knowing, always advanced in the literature.

The remainder of this paper is structured as follows. Section 2 describes the role of technical risk in real options analysis and its description using a continuous time Markov chain. In particular, we analyze also the scenario in which the decision times are dependent on the Markov chains describing the technical risk process.

Section 3 propose some numerical analysis and the comparison of our methodology with Cassimon et al. (2011) approach. Finally, Sect. 4 concludes. 


\section{Compound real option models: the role of technical risk}

In this section, we first provide a short description of the approach proposed by Cassimon et al. (2011) for the valuation of a n-fold compound real option. We illustrate informally the main idea and features of the valuation procedure. Subsequently, we advance our generalization which allows a more efficient and general description of the technical risk process using a continuous-time Markov chain. Theoretical and applied implications of this choice are analyzed and discussed also in relation to previous research articles.

\subsection{Technical risk process with independent success events}

We recall that, in a scheme of n-compound options, the assumptions on which the (Cassimon et al. 2011) model are based foresee that there are two sources of risk: the commercial risk and the technical risk. The commercial risk is related to the normal business activity of the considered company and is usually measured by the volatility of the project return. The technical risk is generally introduced to incorporates uncertainty in the development of the project where each phase can result in a success or not. These two risks need to be modelled and Cassimon et al. (2011) advance two basic hypotheses:

A1: The technical risk process is characterized by success probabilities $p_{i}$ to several phases $i=1, \ldots, n$. These probabilities are assumed to be generated independently from each other and also independently of the commercial risk. Furthermore, the completion times of each phase of the project are assumed to be known.

A2 The commercial risk is described by the Black and Scholes formula. Thus, a no-arbitrage system with two assets is assumed. One asset is risk-free and evolves according to the dynamic

$$
d B_{t}=r B_{t} d t, \quad B_{0}=b
$$

where $r$ is the so-called risk-free rate of interest.

The second asset is a risky one and evolves according to the dynamic

$$
d V_{t}=\mu V_{t} d t+\sigma V_{t} d W_{t}, \quad V_{0}=v,
$$

where $W_{t}$ is a standard Brownian motion.

Consequently, the set of assumptions underlying the Black and Scholes model are assumed to be true.

\subsection{Technical risk process with Markov chain dynamic}

Assumption A1 may be unrealistic in many situations, pharmaceutical R\&D included. The major reason of inadequacy resides in the excessive simplifying idea behind the technical risk process. For this reason, we replace the unrealistic assumption $\mathbf{A 1}$ with a more general and flexible hypothesis: 
A3: The technical risk process is described by a continuous-time Markov chain $\{X(t)\}_{t \in \mathbb{R}}$ with a finite state space $E=\{1, \ldots, m\}$ and generator matrix $\mathbf{A}=$ $\left(a_{i j}\right)_{i, j \in E}$. We recall that

$$
a_{i j}=\lim _{t \rightarrow 0} \frac{p_{i j}(t)-\delta_{i j}}{t} \quad \forall i, j \in E
$$

where

$$
p_{i j}(t)=\mathbb{P}(X(t)=j \mid X(0)=i),
$$

expresses the transition probability function and $\delta_{i j}=\left\{\begin{array}{ll}1 & \text { if } i=j \\ 0 & \text { if } i \neq j\end{array}\right.$ is the Kronecker delta. Obviously, it results that $a_{i i}=-\sum_{j \neq i} a_{i j}$.

Let us consider a n-fold compound option whose vector of known maturities is $\mathbf{T}_{1}^{n}=\left(t_{1}, t_{2}, \ldots, t_{n}\right)$ and corresponding exercise prices $\mathbf{K}_{1}^{n}=\left(K_{1}, K_{2}, \ldots, K_{n}\right)$. At every instant $t_{i}$, we must decide whether to make and continue the investment or abandon it. About that, we define the subset of successful states at $i$ th stage:

$$
U_{t_{i}}:=\{x \in E \text { such that the phase } \mathrm{i} \text { is successful }\} .
$$

Thus, we state that if $X\left(t_{1}\right) \in U_{t_{1}}$, then the first phase is passed and we know also the degree of acceptance of this phase which is expressed by the given value assumed by the Markov chain, i.e. $X\left(t_{1}\right)$. This means that we can consider an ordering relation on the state space of the Markov chain which rates state $i$ as better in term of degree of acceptance of the phase of the project as compared to state $j$ whenever $i$ is lower than $j$, i.e. $i<j$.

Similarly, if $X\left(t_{2}\right) \in U_{t_{2}}$, then the second phase of the project is passed and so on for the remaining phases. Hence, we say that the project has a complete success if and only if

$$
X\left(t_{i}\right) \in U_{t_{i}} \quad \forall i=1, \ldots, n,
$$

and then it is launched in the market.

For instance, consider a two-fold compound option and a Markov chain $\{X(t)\}_{t \in \mathbb{R}}$ with state space $E=\{1, \ldots, 5\}$ to describe the technical risk of the project. Assume that $U_{t_{1}}=\{1,2\}$ and $U_{t_{2}}=\{1\}$. This means that the first phase is realized with an acceptable success if the Markov chain is either in state 1 or 2 , being state 1 preferred to state 2. Consequently, the second phase is successful if and only if $X\left(t_{2}\right)=1$.

Unfortunately, in several real life problems, the vector of decision times $\mathbf{T}_{1}^{n}=$ $\left(t_{1}, t_{2}, \ldots, t_{n}\right)$ cannot be considered deterministic and known in advance. This point is also highlighted, although only at a descriptive level, in Cassimon et al. (2011). There, the authors affirm that a Poisson approach can be used when the technical process does not follow predefined and predictable paths. As stated by the authors, this approach assumes technical probability of success and failure to be constant along the life of the project, the latter being a strong limitation. To overcome this problem we advance the 
use of a random vector of decision times $\mathbf{T}_{1}^{n}=\left(T_{1}, T_{2}, \ldots, T_{n}\right)$ which is dependent on the Markov chains describing the technical risk process. This idea is formalized in the next assumption:

A4: The set of random decision times $\mathbf{T}_{1}^{n}=\left(T_{1}, T_{2}, \cdots, T_{n}\right)$ is a random vector such that:

(i) $T_{k}<T_{k+1} \quad$ a.s. with $T_{n} \leq \tilde{t} \in \mathbb{R}$;

(ii) Denoted by $L_{k}:=T_{k}-T_{k-1}$, for $k=1, \ldots, n$ with $T_{0}=0$ we have that

$$
\begin{aligned}
& \mathbb{P}\left(L_{k} \leq l_{k} \mid T_{k-1}, T_{k-2}, \ldots, T_{1}, T_{0}, X\left(T_{k-1}\right), \ldots, X\left(T_{1}\right), X\left(T_{0}\right)\right) \\
& \quad=\mathbb{P}\left(L_{k} \leq l_{k} \mid X\left(T_{k-1}\right)\right)=F_{X\left(T_{k-1}\right)}\left(l_{k}\right) . \text { a.s. }
\end{aligned}
$$

First, assumption A4 asserts that the total duration of the project is bounded by a real number $\tilde{t}$. It represents the maximum time the investor is available to wait for the successful completion of the project. This is a reasonable choice due to the investor's willingness to get profits within a planned horizon time. Second, the probability distribution function of next investment decision time, which is related to the subsequent phase, depends only on the state of the technical risk process in correspondence of the last decision time. It is worth mentioning that $\mathrm{A4}$ makes the sequence of random times $\left\{T_{i}\right\}_{i=1}^{n}$ dependent on the technical risk process.

In specific applications, or in view of a model simplification, it would be possible to consider the particular case when

$$
F_{i}(\cdot)=F_{j}(\cdot), \forall i \neq j, i, j \in E .
$$

In this situation, the random decision times are independent on the technical risk process in the sense that they do not change according to the states of the technical risk expressed by the Markov chain $\{X(t), t \geq 0\}$.

Let $C_{j_{0}, 0}^{(1 ; n)}(V ; \tilde{t})$ be the current value (at time 0 ) of the most outer call of a n-fold compound option with technical risk process initially valued $j_{0}$, random decision times $\mathbf{T}_{1}^{n}$ not exceeding time $\tilde{t}$ and value of the project denoted by $V$. Recursively, we will denote by $C_{X\left(t_{l}\right), t_{l}}^{(l+1 ; n)}(V ; \tilde{t})$ the value (at time $\left.t_{l}\right)$ of the compound call option on the option $C^{(l+2 ; n)}$ with technical risk process valued $X\left(t_{l}\right)$ with random decision times $\mathbf{T}_{l+1}^{n}$ not exceeding time $\tilde{t}$ and value of the project denoted by $V$.

Before presenting the main result of this paper we provide an important definition:

Definition 1 Fix the horizon time $\tilde{t} \in \mathbb{R}$. A sequence of times and states $\left(\mathbf{t}_{1}^{n}, \mathbf{j}_{1}^{n}\right)$ is called viable if the following conditions are fulfilled:

(i) $t_{i} \leq t_{i+1}, \forall i=1,2, \ldots, n-1$ and $\sum_{i=1}^{n} t_{i} \leq \tilde{t}$;

(ii) $j_{i} \in U_{t_{i}}, \forall i=1, \ldots, n$.

According to this definition, a viable sequence consists of a collection of times (maturities) where the project passes the stages. 
Proposition 1 Under assumptions A2-A4, the value of a $n$-fold compound option with technical risk obeys the following relation:

$$
\begin{aligned}
& C_{j_{0}, 0}^{(1 ; n)}(V, \tilde{t}) \\
& =\int_{0}^{\tilde{t}} \int_{0}^{\tilde{t}-l_{1}} \cdots \int_{0}^{\tilde{t}-\sum_{s=1}^{n-1} l_{s}} \sum_{J_{1}^{n}} \prod_{k=1}^{n} d l_{k}\left(e^{l_{k} \mathbf{A}}\right)_{j_{k-1}, j_{k}} \cdot f_{j_{k-1}}\left(l_{k}\right) \cdot V \\
& \quad \cdot N_{n}\left(a_{1}\left(l_{1}, j_{1}\right), \ldots, a_{n}\left(\sum_{s=1}^{n} l_{s}, j_{n}\right) ; \mathbf{R}_{1}^{n}\right) \\
& \quad-\sum_{m=1}^{n} \int_{0}^{\tilde{t}} \int_{0}^{\tilde{t}-l_{1}} \cdots \int_{0}^{\tilde{t}-\sum_{s=1}^{m-1} l_{s}} \sum_{J_{1}^{m}}^{m} \prod_{k=1}^{m} d l_{k}\left(e^{l_{k} \mathbf{A}}\right)_{j_{k-1}, j_{k}} \cdot f_{j_{k-1}}\left(l_{k}\right) \cdot K_{m} e^{-\sum_{s=1}^{m} l_{s} r} \\
& \quad \cdot N_{m}\left(b_{1}\left(l_{1}, j_{1}\right), \ldots, b_{m}\left(\sum_{s=1}^{m} l_{s}, j_{m}\right) ; \mathbf{R}_{1}^{m}\right)
\end{aligned}
$$

where the notation $\sum_{J_{1}^{k}}=\sum_{j_{1} \in U_{t_{1}}} \sum_{j_{2} \in U_{t_{2}}} \cdots \sum_{j_{k} \in U_{t_{k}}}$ is introduced.

Additionally, set $t_{s}=\sum_{k=1}^{s} l_{k}, \forall s=1, \ldots, n$, we have

$$
\begin{aligned}
& a_{l}\left(t_{l}, j_{l}\right)=b_{l}\left(t_{l}, j_{l}\right)+\sigma \sqrt{t_{l}-t_{0}}, \quad l=1, \ldots, n ; \\
& b_{l}\left(t_{l}, j_{l}\right)=\frac{\ln \left(\frac{V}{\bar{V}_{l}\left(j_{l}\right)}\right)+\left(r-\frac{\sigma^{2}}{2}\right)\left(t_{l}-t_{0}\right)}{\sigma \sqrt{t_{l}-t_{0}}}, \quad l=1, \ldots, n ;
\end{aligned}
$$

$\bar{V}_{l}\left(j_{l}\right)$ is the solution with respect to $V$ of the equation

$$
\begin{aligned}
& C_{j_{l}, t_{l}}^{(l+1 ; n)}(V, \tilde{t})=K_{l}, \text { for } l=1, \ldots, n-1 \text { and } \bar{V}_{n}\left(j_{n}\right)=K_{n}, \forall j_{n} \in U_{t_{n}}, \\
& \rho_{i j}=\sqrt{\frac{t_{i}-t_{0}}{t_{j}-t_{0}}, \quad i<j} \\
& \mathbf{R}_{1}^{l}=\left(r_{i j}^{(l)}\right)_{i, j=1, \ldots, l}, \quad r_{i j}^{(l)}= \begin{cases}1 & \text { if } i=j \\
\rho_{i j} & \text { if } i<j \\
\rho_{j i} & \text { if } i>j\end{cases}
\end{aligned}
$$

Proof The price of an option can be expressed as the difference between the expected present value of the underlying minus the expected present value of the exercise price given the option is in the money.

Observe that, in our framework, the option can be in the money only if there is a success at every phase of the project. This holds if

$$
X\left(T_{i}\right) \in U_{T_{i}}, \forall i=1, \ldots, n .
$$

Assume, momentarily, to deal with a standard n-fold compound option with known maturities $\mathbf{t}_{1}^{n}=\left(t_{1}, t_{2}, \cdots, t_{n}\right)$ and no technical risk. According to Carr (1988) and 
Thomassen et al. (2002), the expected present value of the underlying given the option is in the money is given by

$$
V \cdot N_{n}\left(a_{1}\left(t_{1}\right), \ldots, a_{n}\left(t_{n}\right) ; \mathbf{R}_{1}^{n}\right),
$$

while the expected present value of the exercise price is

$$
\sum_{m=1}^{n} K_{m} e^{-r\left(t_{m}-t_{0}\right)} \cdot N_{m}\left(b_{1}\left(t_{1}\right), \ldots, b_{m}\left(t_{m}\right) ; \mathbf{R}_{1}^{m}\right),
$$

where

$$
\begin{aligned}
& a_{l}\left(t_{l}\right)=b_{l}\left(t_{l}\right)+\sigma \sqrt{t_{l}-t_{0}}, \quad l=1, \ldots, n ; \\
& b_{l}\left(t_{l}\right)=\frac{\ln \left(\frac{V}{\bar{V}_{l}}\right)+\left(r-\frac{\sigma^{2}}{2}\right)\left(t_{l}-t_{0}\right)}{\sigma \sqrt{t_{l}-t_{0}}}, \quad l=1, \ldots, n ;
\end{aligned}
$$

$\bar{V}_{l}$ is the solution with respect to $V$ of the equation

$$
\begin{aligned}
& C_{t_{l}}^{(l+1 ; n)}\left(V ; \mathbf{t}_{l+1}^{n}, \tilde{t}\right)=K_{l}, \text { for } l=1, \ldots, n-1 \text { and } \bar{V}_{n}=K_{n} \\
& \rho_{i j}=\sqrt{\frac{t_{i}-t_{0}}{t_{j}-t_{0}}, \quad i<j,} \\
& \mathbf{R}_{1}^{l}=\left(r_{i j}^{(l)}\right)_{i, j=1, \ldots, l}, \quad r_{i j}^{(l)}= \begin{cases}1 & \text { if } i=j \\
\rho_{i j} & \text { if } i<j \\
\rho_{j i} & \text { if } i>j\end{cases}
\end{aligned}
$$

In our more general framework, the expected values (4) and (5) depend on the value assumed by the random vector $\mathbf{T}_{0}^{n}$ and on those of the corresponding technical risk process $X\left(\mathbf{T}_{0}^{n}\right)$. Thus, let $\mathcal{R}: \mathbb{R}^{(n+1)} \times E^{(n+1)} \times \mathbb{R} \longrightarrow \mathbb{R}$ be a function defined according to the relation

$$
\begin{aligned}
\mathcal{R}\left(\mathbf{t}_{0}^{n}, \mathbf{j}_{0}^{n}, \tilde{t}\right):=V & \cdot N_{n}\left(a_{1}\left(t_{1}, j_{1}\right), \ldots, a_{n}\left(t_{n}, j_{n}\right) ; \mathbf{R}_{1}^{n}\right) \chi\left(j_{i} \in U_{t_{i}},\right. \\
& \left.\forall i=1, \ldots, n, t_{n} \leq \bar{t}\right) \\
- & \sum_{m=1}^{n} K_{m} e^{-r\left(t_{m}-t_{0}\right)} \cdot N_{m}\left(b_{1}\left(t_{1}, j_{1}\right), \ldots, b_{m}\left(t_{m}, j_{m}\right) ; \mathbf{R}_{1}^{m}\right) \\
\cdot & \chi\left(j_{i} \in U_{t_{i}}, \forall i=1, \ldots, m, t_{m} \leq \bar{t}\right) .
\end{aligned}
$$

Formula (9) expresses the value of a n-fold compound option with viable technical risk trajectory $\left(\mathbf{t}_{1}^{n}, \mathbf{j}_{1}^{n}\right)$.

A relevant difference characterizes our framework from that analyzed in Cassimon et al. (2011) and relies on the need to modify the boundary conditions along any viable technical risk trajectory $\left(\mathbf{t}_{1}^{n}, \mathbf{j}_{1}^{n}\right)$. Indeed, in our setting the technical success probabilities are not independent and then, the value of the inner compound options 
depend on the specific state occupied by the technical process and completion times of the phases. Thus, the new boundary conditions are:

$$
C_{j_{i}, t_{i}}^{(i ; n)}(V, \tilde{t})=\max \left(0, C_{j_{i+1}, t_{i+1}}^{(i+1 ; n)}(V, \tilde{t})-K_{i}\right), \forall i=1, \ldots, n-1,
$$

and

$$
C_{j_{n}, t_{n}}^{(n ; n)}(V, \tilde{t})=\max \left(0, V-K_{n}\right) .
$$

Equation (11) just says that, conditional on the success of the project, the payoff is given by the positive difference between the value of the project at time $t_{n}$ and the strike price $K_{n}$, thus the most outer call is exercised only if $V\left(t_{n}\right)>K_{n}$ (conditionally to a viable technical trajectory).

As regard to relation (10) we observe that the boundary conditions vary depending on the specific trajectory of the technical process because the value of the (i+1)-fold call option depends both on the state $j_{i+1}$ of the technical risk process and on the time $t_{i+1}$. Precisely, this is due to different probabilities of success in next phase according to the state $j_{i+1}$ of the Markov chain and also to the time $t_{i+1}$ through the distribution of the random variable $T_{i+2}$ and the constraint $T_{n} \leq \tilde{t}$.

Accordingly, the values of the project that identify the exercising or not exercising actions depend on the trajectory and are denoted by $\bar{V}_{l}\left(j_{l}, t_{l}\right)$ which are defined as those values of the project that solve the equations

$C_{j_{l}, t_{l}}^{(l ; n)}(V, \tilde{t})=K_{l-1}$, for $l=2, \ldots, n-1$ and $\tilde{V}_{n}\left(j_{n}, t_{n}\right)=K_{n}, \forall j_{n} \in U_{t_{n}}, t_{n} \leq \tilde{t}$

Thus, in turn equation (7) updates to

$$
b_{l}\left(j_{l}, t_{l}\right)=\frac{\ln \left(\frac{V}{\tilde{V}_{l}\left(j_{l}, t_{l}\right)}\right)+\left(r-\frac{\sigma^{2}}{2}\right)\left(t_{l}-t_{0}\right)}{\sigma \sqrt{t_{l}-t_{0}}}, \quad l=1, \ldots, n ;
$$

and equation (6) becomes

$$
a_{l}\left(j_{l}, t_{l}\right)=b_{l}\left(j_{l}, t_{l}\right)+\sigma \sqrt{t_{l}-t_{0}}, \quad l=1, \ldots, n .
$$

Now, we can consider the random variable $\mathcal{R}\left(\mathbf{T}_{0}^{n}, X\left(\mathbf{T}_{0}^{n}\right), \tilde{t}\right)$ as a functional of the technical risk process $\left(\mathbf{T}_{0}^{n}, X(t), t \geq 0\right)$ and we can express the price of the $n$-fold compound option conditional on some initial conditions, say

$$
T_{0}=0, X\left(T_{0}\right)=j_{0} \in E,
$$

according to

$$
C_{j_{0}, 0}^{(1 ; n)}(V, \tilde{t})=\mathbb{E}\left[\mathcal{R}\left(\mathbf{T}_{0}^{n}, X\left(\mathbf{T}_{0}^{n}\right), \tilde{t}\right) \mid T_{0}=0, X\left(T_{0}\right)=j_{0}\right]
$$

Let us proceed now to the computation of (15) under the assumptions A2-A4. To this end, define $L_{i}=T_{i}-T_{i-1}, \forall i=1, \ldots, n$ and integrate over all possible values of 
the random vectors $\left(L_{1}, \ldots, L_{n}\right)$ and $\left(X\left(T_{1}\right), \ldots, X\left(T_{n}\right)\right)$ to get

$$
\begin{aligned}
& C_{j_{0}, 0}^{(1 ; n)}(V, \tilde{t}) \\
& =\int_{0}^{\infty} \cdots \int_{0}^{\infty} \sum_{j_{1} \in E} \cdots \sum_{j_{n} \in E} \mathbb{P}\left[L_{1} \in\left(l_{1}, l_{1}+d l_{1}\right),\right. \\
& \left.\ldots, L_{n} \in\left(l_{n}, l_{n}+d l_{n}\right), X\left(l_{1}\right)=j_{1}, \ldots, X\left(\sum_{s=1}^{n} l_{s}\right)=j_{n}\right] \\
& \cdot\left\{V \cdot N_{n}\left(a_{1}\left(l_{1}, j_{1}\right), \ldots, a_{n}\left(\sum_{s=1}^{n} l_{s}, j_{n}\right) ; \mathbf{R}_{1}^{n}\right)\right. \\
& \chi\left(X\left(\sum_{s=1}^{i} l_{s}\right) \in U_{\sum_{s=1}^{i} l_{s}}, \forall i=1, \ldots, n, t_{n} \leq \tilde{t}\right) \\
& -\sum_{m=1}^{n} K_{m} e^{-\sum_{s=1}^{m} l_{s} r} \cdot N_{m}\left(b_{1}\left(l_{1}, j_{1}\right), \ldots, b_{m}\left(\sum_{s=1}^{m} l_{s}, j_{m}\right) ; \mathbf{R}_{1}^{m}\right) \\
& \left.\chi\left(X\left(\sum_{s=1}^{i} l_{s}\right) \in U_{\sum_{s=1}^{i} l_{s}}, \forall i=1, \ldots, m, t_{m} \leq \tilde{t}\right)\right\} \\
& =\int_{0}^{\infty} \cdots \int_{0}^{\infty} \sum_{j_{1} \in E} \cdots \sum_{j_{n} \in E} \mathbb{P}\left[L_{1} \in\left(l_{1}, l_{1}+d l_{1}\right),\right. \\
& \left.\ldots, L_{n} \in\left(l_{n}, l_{n}+d l_{n}\right), X\left(l_{1}\right)=j_{1}, \ldots, X\left(\sum_{s=1}^{n} l_{s}\right)=j_{n}\right] \\
& \cdot V \cdot N_{n}\left(a_{1}\left(l_{1}, j_{1}\right), \ldots, a_{n}\left(\sum_{s=1}^{n} l_{s}, j_{n}\right) ; \mathbf{R}_{1}^{n}\right) \\
& \chi\left(X\left(\sum_{s=1}^{i} l_{s}\right) \in U_{\sum_{s=1}^{i} l_{s}}, \forall i=1, \ldots, n, t_{n} \leq \tilde{t}\right) \\
& -\sum_{m=1}^{n} \int_{0}^{\infty} \cdots \int_{0}^{\infty} \sum_{j_{1} \in E} \cdots \sum_{j_{n} \in E} \mathbb{P}\left[L_{1} \in\left(l_{1}, l_{1}+d l_{1}\right),\right. \\
& \left.\ldots, L_{n} \in\left(l_{n}, l_{n}+d l_{n}\right), X\left(l_{1}\right)=j_{1}, \ldots, X\left(\sum_{s=1}^{n} l_{s}\right)=j_{n}\right] \\
& \cdot K_{m} e^{-\sum_{s=1}^{m} l_{s} r} \cdot N_{m}\left(b_{1}\left(l_{1}, j_{1}\right), \ldots, b_{m}\left(\sum_{s=1}^{m} l_{s}, j_{m}\right) ; \mathbf{R}_{1}^{m}\right) \\
& \left.\chi\left(X\left(\sum_{s=1}^{i} l_{s}\right) \in U_{\sum_{s=1}^{i} l_{s}}, \forall i=1, \ldots, m, t_{m} \leq \tilde{t}\right)\right\} \\
& =: I_{1}+I_{2} \text {, }
\end{aligned}
$$


having denoted by $I_{1}$ and $I_{2}$ the two previous multiple integrals.

Now, by repeated applications of assumptions A3 and A4, we have

$$
\begin{aligned}
& I_{1}=\int_{0}^{\infty} \cdots \int_{0}^{\infty} \sum_{j_{1} \in E} \cdots \sum_{j_{n} \in E} \mathbb{P}\left[X\left(\sum_{s=1}^{n} l_{s}\right)=j_{n} \mid T_{0}=0\right. \\
& L_{1}=l_{1}, \ldots, L_{n}=l_{n}, X(0)=j_{0}, \\
& \left.X\left(l_{1}\right)=j_{1}, \ldots, X\left(\sum_{s=1}^{n-1} l_{s}\right)=j_{n-1}\right] \cdot \mathbb{P}\left[L_{n} \in\left(l_{n}, l_{n}+d l_{n}\right) \mid T_{0}=0,\right. \\
& L_{1} \in\left(l_{1}, l_{1}+d l_{1}\right), \ldots, \\
& L_{n-1} \in\left(l_{n-1}, l_{n-1}+d l_{n-1}\right), X(0)=j_{0}, X\left(l_{1}\right)=j_{1} \text {, } \\
& \left.\ldots, X\left(\sum_{s=1}^{n-1} l_{s}\right)=j_{n-1}\right] \text {. } \\
& \ldots \cdot \mathbb{P}\left[X\left(l_{1}\right)=j_{1} \mid T_{0}=0, L_{1}=l_{1}, X(0)=j_{0}\right] \\
& \text {. } \mathbb{P}\left[L_{1} \in\left(l_{1}, l_{1}+d l_{1}\right) \mid T_{0}=0, X(0)=j_{0}\right] \\
& \cdot V \cdot N_{n}\left(a_{1}\left(l_{1}, j_{1}\right), \ldots, a_{n}\left(\sum_{s=1}^{n} l_{s}, j_{n}\right) ; \mathbf{R}_{1}^{n}\right) \chi\left(X\left(\sum_{s=1}^{i} l_{s}\right)\right. \\
& \left.\in U_{\sum_{s=1}^{i} l_{s}}, \forall i=1, \ldots, n, t_{n} \leq \tilde{t}\right) \\
& =\int_{0}^{\infty} \cdots \int_{0}^{\infty} \sum_{j_{1} \in E} \cdots \sum_{j_{n} \in E} \mathbb{P}\left[X\left(\sum_{s=1}^{n} l_{s}\right)=j_{n} \mid X\left(\sum_{s=1}^{n-1} l_{s}\right)=j_{n-1}\right] \\
& \cdot \mathbb{P}\left[L_{n} \in\left(l_{n}, l_{n}+d l_{n}\right) \mid X\left(\sum_{s=1}^{n-1} l_{s}\right)=j_{n-1}\right] \\
& \ldots \cdot \mathbb{P}\left[X\left(l_{1}\right)=j_{1} \mid X(0)=j_{0}\right] \cdot \mathbb{P}\left[L_{1} \in\left(l_{1}, l_{1}+d l_{1}\right) \mid X(0)=j_{0}\right] \cdot V \\
& \cdot N_{n}\left(a_{1}\left(l_{1}, j_{1}\right), \ldots, a_{n}\left(\sum_{s=1}^{n} l_{s}, j_{n}\right) ; \mathbf{R}_{1}^{n}\right) \\
& \cdot \chi\left(X\left(\sum_{s=1}^{i} l_{s}\right) \in U_{\sum_{s=1}^{i} l_{s}}, \forall i=1, \ldots, n, t_{n} \leq \tilde{t}\right) \\
& =\int_{0}^{\infty} \cdots \int_{0}^{\infty} \sum_{j_{1} \in E} \cdots \sum_{j_{n} \in E} \mathbb{P}\left[X\left(l_{n}\right)=j_{n} \mid X(0)=j_{n-1}\right] . \\
& \mathbb{P}\left[L_{n} \in\left(l_{n}, l_{n}+d l_{n}\right) \mid X\left(\sum_{s=1}^{n-1} l_{s}\right)=j_{n-1}\right] \\
& \ldots \cdot \mathbb{P}\left[X\left(l_{1}\right)=j_{1} \mid X(0)=j_{0}\right] \cdot \mathbb{P}\left[L_{1} \in\left(l_{1}, l_{1}+d l_{1}\right) \mid X(0)=j_{0}\right] \cdot \\
& V \cdot N_{n}\left(a_{1}\left(l_{1}, j_{1}\right), \ldots, a_{n}\left(\sum_{s=1}^{n} l_{s}, j_{n}\right) ; \mathbf{R}_{1}^{n}\right)
\end{aligned}
$$




$$
\cdot \chi\left(X\left(\sum_{s=1}^{i} l_{s}\right) \in U_{\sum_{s=1}^{i} l_{s}}, \forall i=1, \ldots, n, t_{n} \leq \tilde{t}\right)
$$

where the latter equality is obtained using the time homogeneity property of the Markov process $(X(t), t \geq 0)$.

The previous multiple integral assumes a non-zero value only when evaluated along any viable trajectory of the technical risk process. Thus, the integration should be limited to all those sample paths such that $\sum_{s=1}^{n} l_{s} \leq \tilde{t}$ which in turn implies that

$$
l_{n-i} \leq \tilde{t}-\sum_{s=1}^{n-(i+1)} l_{s}, \quad \forall i=1, \ldots, n-1,
$$

with the convention that $\sum_{s=1}^{0} l_{s}=0$. Furthermore, observe that viable trajectories make provision for the belonginess of the Markov process $(X(t), t \geq 0)$ to the successful states at each stage of the project, i.e.

$$
X\left(\sum_{s=1}^{i} l_{s}\right) \in U_{\sum_{s=1}^{i} l_{s}}, \quad \forall i=1, \ldots, n .
$$

Hence, once the abbreviate notation

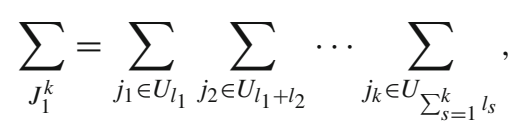

is introduced, we can use the exponential representation of the transition probability function of the Markov process and the definition of the density function on the conditional distribution of (1) to get

$$
\begin{aligned}
I_{1} & =\int_{0}^{\tilde{t}} \int_{0}^{\tilde{t}-l_{1}} \cdots \int_{0}^{\tilde{t}-\sum_{s=1}^{n-1} l_{s}} \sum_{J_{1}^{n}} \prod_{k=1}^{n} d l_{k}\left(e^{l_{k} \mathbf{A}}\right)_{j_{k-1}, j_{k}} \cdot f_{j_{k-1}}\left(l_{k}\right) \cdot V \\
& \cdot N_{n}\left(a_{1}\left(l_{1}, j_{1}\right), \ldots, a_{n}\left(\sum_{s=1}^{n} l_{s}, j_{n}\right) ; \mathbf{R}_{1}^{n}\right) .
\end{aligned}
$$

The computation of the integral $I_{2}$ shares similar ideas as those underlying the integral $I_{1}$. Indeed, we have 


$$
\begin{aligned}
I_{2}= & -\sum_{m=1}^{n} \int_{0}^{\infty} \ldots \int_{0}^{\infty} \sum_{j_{1} \in E} \\
& \ldots \sum_{j_{n} \in E} \mathbb{P}\left[L_{1} \in\left(l_{1}, l_{1}+d l_{1}\right), \ldots, L_{n} \in\left(l_{n}, l_{n}+d l_{n}\right), X\left(l_{1}\right)=j_{1},\right. \\
& \left.\ldots, X\left(\sum_{s=1}^{n} l_{s}\right)=j_{n}\right] \\
& \cdot K_{m} e^{-\sum_{s=1}^{m} l_{s} r} \cdot N_{m}\left(b_{1}\left(l_{1}, j_{1}\right), \ldots, b_{m}\left(\sum_{s=1}^{m} l_{s}, j_{m}\right) ; \mathbf{R}_{1}^{m}\right) \chi\left(X\left(\sum_{s=1}^{i} l_{s}\right)\right. \\
\in & \left.U_{\sum_{s=1}^{i} l_{s}}, \forall i=1, \ldots, m, t_{m} \leq \tilde{t}\right)
\end{aligned}
$$

Repeated applications of assumptions A3 and A4 produce

$$
\begin{aligned}
I_{2}= & -\sum_{m=1}^{n} \int_{0}^{\infty} \cdots \int_{0}^{\infty} \sum_{j_{1} \in E} \cdots \sum_{j_{n} \in E} \mathbb{P}\left[X\left(\sum_{s=1}^{n} l_{s}\right)=j_{n} \mid X\left(\sum_{s=1}^{n-1} l_{s}\right)=j_{n-1}\right] \\
& \cdot \mathbb{P}\left[L_{n} \in\left(l_{n}, l_{n}+d l_{n}\right) \mid X\left(\sum_{s=1}^{n-1} l_{s}\right)=j_{n-1}\right] \cdot \ldots \cdot \mathbb{P}\left[X\left(l_{1}\right)=j_{1} \mid X(0)=j_{0}\right] \\
& \cdot \mathbb{P}\left[L_{1} \in\left(l_{1}, l_{1}+d l_{1}\right) \mid X(0)=j_{0}\right] \cdot K_{m} e^{-\sum_{s=1}^{m} l_{s} r} \\
& \cdot N_{m}\left(b_{1}\left(l_{1}, j_{1}\right), \ldots, b_{m}\left(\sum_{s=1}^{m} l_{s}, j_{m}\right) ; \mathbf{R}_{1}^{m}\right) \\
& \cdot \chi\left(X\left(\sum_{s=1}^{i} l_{s}\right) \in U_{\sum_{s=1}^{i} l_{s}}, \forall i=1, \ldots, m, t_{m} \leq \tilde{t}\right)
\end{aligned}
$$

Observe now that

$$
\sum_{j_{n} \in E} \mathbb{P}\left[X\left(\sum_{s=1}^{n} l_{s}\right)=j_{n} \mid X\left(\sum_{s=1}^{n-1} l_{s}\right)=j_{n-1}\right]=1
$$

and in turn

$$
\int_{0}^{+\infty} \mathbb{P}\left[L_{n} \in\left(l_{n}, l_{n}+d l_{n}\right) \mid X\left(\sum_{s=1}^{n-1} l_{s}\right)=j_{n-1}\right]=1 .
$$


Repeat the same considerations as in (18) and (19) for all stages $i>m$ to get

$$
\begin{aligned}
I_{2}= & -\sum_{m=1}^{n} \int_{0}^{\infty} \cdots \int_{0}^{\infty} \sum_{j_{1} \in E} \cdots \sum_{j_{m} \in E} \mathbb{P}\left[X\left(\sum_{s=1}^{m} l_{s}\right)=j_{m} \mid X\left(\sum_{s=1}^{m-1} l_{s}\right)=j_{m-1}\right] \\
& \cdot \mathbb{P}\left[L_{m} \in\left(l_{m}, l_{m}+d l_{m}\right) \mid X\left(\sum_{s=1}^{m-1} l_{s}\right)=j_{m-1}\right] \ldots \cdot \mathbb{P}\left[X\left(l_{1}\right)=j_{1} \mid X(0)=j_{0}\right] \\
& \cdot \mathbb{P}\left[L_{1} \in\left(l_{1}, l_{1}+d l_{1}\right) \mid X(0)=j_{0}\right] \cdot K_{m} e^{-\sum_{s=1}^{m} l_{s} r} \\
& \cdot N_{m}\left(b_{1}\left(l_{1}, j_{1}\right), \ldots, b_{m}\left(\sum_{s=1}^{m} l_{s}, j_{m}\right) ; \mathbf{R}_{1}^{m}\right) \\
& \cdot \chi\left(X\left(\sum_{s=1}^{i} l_{s}\right) \in U_{\sum_{s=1}^{i} l_{s}}, \forall i=1, \ldots, m, t_{m} \leq \tilde{t}\right) .
\end{aligned}
$$

Now, the same arguments used for the computation of the integral $I_{1}$ give

$$
\begin{aligned}
I_{2} & =-\sum_{m=1}^{n} \int_{0}^{\tilde{t}} \int_{0}^{\tilde{t}-l_{1}} \cdots \int_{0}^{\tilde{t}-\sum_{s=1}^{m-1} l_{s}} \sum_{J_{1}^{m}} \prod_{k=1}^{m} d l_{k}\left(e^{l_{k} \mathbf{A}}\right)_{j_{k-1}, j_{k}} \cdot f_{j_{k-1}}\left(l_{k}\right) \cdot K_{m} e^{-\sum_{s=1}^{m} l_{s} r} \\
& \cdot N_{m}\left(b_{1}\left(l_{1}, j_{1}\right), \ldots, b_{m}\left(\sum_{s=1}^{m} l_{s}, j_{m}\right) ; \mathbf{R}_{1}^{m}\right) .
\end{aligned}
$$

This completes the proof.

Formula (2) is very general and includes, as particular cases, simpler results, some of them already presented in literature. Therefore, it may be useful to discuss some of them briefly.

\subsubsection{Case with unbounded horizon time}

Let us consider the case when the investor does not have any upper bound to the total duration of the project. The price of the option can be obtained considering

$$
C_{j_{0}, 0}^{(1 ; n)}(V)=\lim _{\bar{t} \rightarrow \infty} C_{j_{0}, 0}^{(1 ; n)}(V, \tilde{t})
$$

Thus, we conclude that

$$
\begin{aligned}
C_{j_{0}, 0}^{(1 ; n)}(V)=\int_{0}^{\infty} & \cdots \int_{0}^{\infty} \sum_{J_{1}^{n}} \prod_{k=1}^{n} d l_{k}\left(e^{l_{k} \mathbf{A}}\right)_{j_{k-1}, j_{k}} \\
& \times \cdot f_{j_{k-1}}\left(l_{k}\right) \cdot V \cdot N_{n}\left(a_{1}\left(l_{1}, j_{1}\right), \ldots, a_{n}\left(\sum_{s=1}^{n} l_{s}, j_{n}\right) ; \mathbf{R}_{1}^{n}\right)
\end{aligned}
$$




$$
\begin{aligned}
& -\sum_{m=1}^{n} \int_{0}^{\infty} \cdots \int_{0}^{\infty} \sum_{J_{1}^{m}} \prod_{k=1}^{m} d l_{k}\left(e^{l_{k} \mathbf{A}}\right)_{j_{k-1}, j_{k}} \cdot f_{j_{k-1}}\left(l_{k}\right) \cdot K_{m} e^{-\sum_{s=1}^{m} l_{s} r} \\
& \cdot N_{m}\left(b_{1}\left(l_{1}, j_{1}\right), \ldots, b_{m}\left(\sum_{s=1}^{m} l_{s}, j_{m}\right) ; \mathbf{R}_{1}^{m}\right) .
\end{aligned}
$$

Equation (20) expresses the value of a n-fold compound option with technical risk described by a Markov chain and random decision times correlated to the Markov process in the case when the project is completed after the success of all the n-phases without any bound to the duration of the contract.

Remark 1 The computation of formulas (2) and (20) is quite complex because it requires integration and summation as well as the solution of the equation (12) over all possible paths (trajectories) of the technical risk process.

Definition 2 Let consider an n-phase investment project with random decision times $\mathbf{T}_{1}^{n}=\left(T_{1}, T_{2}, \ldots, T_{n}\right)$ and unbounded horizon time. Let $X(0)=j_{0}$ be the value of the technical risk process at the beginning of the project. The conditional success probability of the first $k$ phases, with $k \in\{1,2, \ldots, n\}$, is defined to be

$$
h_{k, n}\left(j_{0}, \mathbf{T}_{1}^{k}\right):=\mathbb{P}\left(X\left(T_{k}\right) \in U_{T_{k}}, \ldots, X\left(T_{1}\right) \in U_{T_{1}} \mid X(0)=j_{0}\right)
$$

Corollary 1 Under assumptions A3-A4, the conditional success probability of the first $k$ phases, with $k \in\{1,2, \ldots, n\}$, are given by

$$
h_{k, n}\left(j_{0}, \mathbf{T}_{1}^{k}\right)=\int_{0}^{\infty} \cdots \int_{0}^{\infty} \sum_{J_{1}^{k}} \prod_{m=1}^{k} d l_{m}\left(e^{l_{m} \mathbf{A}}\right)_{j_{m-1}, j_{m}} \cdot f_{j_{m-1}}\left(l_{m}\right)
$$

Proof We only observe that in the proof of Proposition 1 we integrated over the joint distribution of the random vectors $\left(L_{1}, \ldots, L_{n}\right)$ and $\left(X\left(T_{1}\right), \ldots, X\left(T_{n}\right)\right)$. A repetition of the previous computation limited to the first $k$ phases gives the result.

\subsubsection{Case with deterministic decision times}

If the vector of maturities $\left\{L_{i}\right\}_{i=1}^{n}$ is a set of degenerate random variables with cumulative distribution functions given $\forall k=1, \ldots, n$ by the Heaviside function

$$
F_{j_{k-1}}\left(l_{k}\right)= \begin{cases}1 & \text { if } l_{k} \geq l_{k}^{*} \\ 0 & \text { if } l_{k}<l_{k}^{*}\end{cases}
$$

then, by substitution of (23) into formula (2) we get next formula (24) which expresses the value of a $n$-fold compound option with deterministic maturities $\mathbf{T}_{1}^{n}=\left(l_{1}^{*}, l_{1}^{*}+\right.$ 


$$
\begin{aligned}
l_{2}^{*}, \cdots, l_{1}^{*}+\ldots+ & \left.l_{n}^{*}\right) \text {. Thus, set } t_{k}=\sum_{s=1}^{k} l_{s}^{*} \text { to get } \\
C_{j_{0}, 0}^{(1 ; n)}\left(V ; \mathbf{t}_{1}^{n}\right)= & \sum_{J_{1}^{n}} \prod_{k=1}^{n}\left(e^{\left(t_{k}-t_{k-1}\right) \mathbf{A}}\right)_{j_{k-1}, j_{k}} \cdot V \cdot N_{n}\left(a_{1}\left(t_{1}, j_{1}\right), \ldots, a_{n}\left(t_{n}, j_{n}\right) ; \mathbf{R}_{1}^{n}\right) \\
& -\sum_{m=1}^{n} \sum_{J_{1}^{m}} \prod_{k=1}^{m}\left(e^{\left(t_{k}-t_{k-1}\right) \mathbf{A}}\right)_{j_{k-1}, j_{k}} \cdot K_{m} e^{-t_{m} r} \\
& \cdot N_{m}\left(b_{1}\left(t_{1}, j_{1}\right), \ldots, b_{m}\left(t_{m}, j_{m}\right) ; \mathbf{R}_{1}^{m}\right) .
\end{aligned}
$$

In the case with deterministic decision times $\mathbf{t}_{1}^{n}=\left(t_{1}, t_{2}, \cdots, t_{n}\right)$, the conditional success probability of the first $k$ phases, with $k \in\{1,2, \ldots, n\}$, is

$$
h_{k, n}\left(j_{0}, \mathbf{t}_{1}^{k}\right):=\mathbb{P}\left(X\left(t_{k}\right) \in U_{t_{k}}, \ldots, X\left(t_{1}\right) \in U_{t_{1}} \mid X(0)=j_{0}\right) .
$$

A simple iterative application of the Markov property gives

$$
h_{k, n}\left(j_{0}, \mathbf{t}_{1}^{k}\right)=\sum_{J_{1}^{k}} \prod_{s=1}^{k} p_{j_{s-1} j_{s}}\left(t_{s}-t_{s-1}\right) \text {, }
$$

where $t_{0}=0$ and $p_{j_{s-1} j_{s}}\left(t_{s}-t_{s-1}\right)=\left(e^{\left(t_{s}-t_{s-1}\right) \mathbf{A}}\right)_{j_{s-1} j_{s}}$.

\subsubsection{The Cassimon et al. (2011) model}

Now let us consider a very particular case when the decision times are deterministic and the technical risk process admits the same transition probabilities independently on the state occupied by the Markov process. This occurs for a generator matrix $\mathbf{A}=\left(a_{i, j}\right)_{i, j \in E}$ such that:

$$
\forall i \neq h, a_{i j}=a_{h j} \forall j \in E
$$

In this case, we may denote by $p(k):=\sum_{j \in U_{t_{k}}}\left(e^{\left(t_{k}-t_{k-1}\right) \mathbf{A}}\right)_{i, j}$ and obtain that $h_{k, n}\left(i, \mathbf{t}_{1}^{k}\right)=h_{k, n}\left(j, \mathbf{t}_{1}^{k}\right)$ for all $i \neq j$ where

$$
h_{k, n}\left(j, \mathbf{t}_{1}^{k}\right)=\prod_{s=1}^{k} p(s) .
$$

In this fashion, we recover exactly the Cassimon et al. (2011) formula, i.e.

$$
\begin{aligned}
C^{(1 ; n)}\left(V ; \mathbf{t}_{1}^{n}\right)= & h_{n ; n}\left(\mathbf{t}_{1}^{n}\right) \cdot V \cdot N_{n}\left(a_{1}\left(t_{1}\right), \ldots, a_{n}\left(t_{n}\right) ; \mathbf{R}_{1}^{n}\right) \\
& -\sum_{m=1}^{n} h_{m ; n}\left(\mathbf{t}_{1}^{m}\right) \cdot K_{m} e^{-t_{m} r} \cdot N_{m}\left(b_{1}\left(t_{1}\right), \ldots, b_{m}\left(t_{m}\right) ; \mathbf{R}_{1}^{m}\right)
\end{aligned}
$$


where

$$
\begin{aligned}
& a_{l}=b_{l}+\sigma \sqrt{t_{l}-t_{0}}, \quad l=1, \ldots, n ; \\
& b_{l}=\frac{\ln \left(\frac{V}{\bar{V}_{l}}\right)+\left(r-\frac{\sigma^{2}}{2}\right)\left(t_{l}-t_{0}\right)}{\sigma \sqrt{t_{l}-t_{0}}}, \quad l=1, \ldots, n ;
\end{aligned}
$$

$\bar{V}_{l}$ is the solution with respect to $V$ of the equation $C^{(l+1 ; n)}\left(V, t_{l}\right)=K_{l}$ for $l=$ $1, \ldots, n-1$ and $\bar{V}_{n}=K_{n}$.

$$
\begin{aligned}
& \rho_{i j}=\sqrt{\frac{t_{i}-t_{0}}{t_{j}-t_{0}}}, \quad i<j \\
& \mathbf{R}_{1}^{l}=\left(r_{i j}^{(l)}\right)_{i, j=1, \ldots, l}, \quad r_{i j}^{(l)}=\left\{\begin{array}{cc}
1 & \text { if } i=j \\
\rho_{i j} & \text { if } i<j \\
\rho_{j i} & \text { if } i>j
\end{array}\right.
\end{aligned}
$$

\subsubsection{The Geske (1979) model}

The last case we consider is when $n=2$ and the technical risk process is such that $h_{i, 2}\left(\mathbf{t}_{1}^{i}\right)=1, \forall i=1,2$. This scenario coincides with the one studied by Geske (1979) about a two-fold compound option.

$$
C=V N_{2}\left(a_{1}, a_{2} ; R^{2}\right)-K_{2} e^{-r\left(t_{2}-t_{0}\right)} N_{2}\left(b_{1}, b_{2} ; R^{2}\right)-K_{1} e^{-r\left(t_{1}-t_{0}\right)} N_{1}\left(b_{1}\right),
$$

where

$$
b_{1}=\frac{\ln \left(\frac{V}{V_{1}}\right)+\left(r-\frac{\sigma^{2}}{2}\right)\left(t_{1}-t_{0}\right)}{\sigma \sqrt{\left(t_{1}-t_{0}\right)}} ; \quad b_{2}=\frac{\ln \left(\frac{V}{K_{2}}\right)+\left(r-\frac{\sigma^{2}}{2}\right)\left(t_{2}-t_{0}\right)}{\sigma \sqrt{\left(t_{2}-t_{0}\right)}}
$$

where

$$
a_{1}=b_{1}+\sigma \sqrt{t_{1}-t_{0}} ; \quad a_{2}=b_{2}+\sigma \sqrt{t_{2}-t_{0}} ; \quad R^{2}=\sqrt{\left(t_{1}-t_{0}\right)\left(t_{2}-t_{0}\right)}
$$

$N_{2}$ is the bivariate cumulative normal distribution function and $\overline{V_{1}}$ is the critical price of $V$ such that:

$$
V N_{1}\left(\overline{a_{1}}\right)-K_{2} e^{-r\left(t_{2}-t_{1}\right)} N_{1}\left(\overline{b_{2}}\right)-K_{1}=0,
$$

with

$$
\overline{b_{2}}=\frac{\ln \left(\frac{V}{K_{2}}\right)+\left(r-\frac{\sigma^{2}}{2}\right)\left(t_{2}-t_{1}\right)}{\sigma \sqrt{\left(t_{2}-t_{1}\right)}} ; \quad \overline{a_{2}}=\overline{b_{2}}+\sigma \sqrt{t_{2}-t_{1}} .
$$




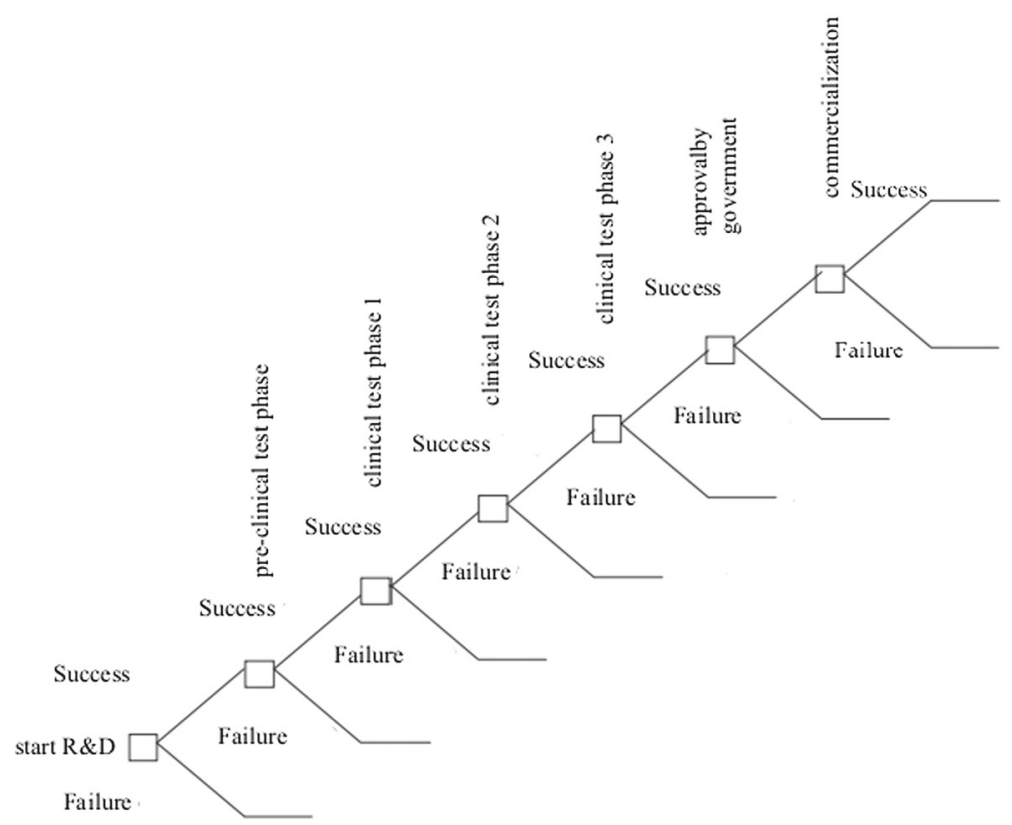

Fig. 1 Structure of an $n$ compound option as described in Cassimon et al. (2011)

\section{Numerical application}

In this section, we propose some numerical applications to highlight the method presented in our paper. An R\&D investment in pharmaceutical sector can be seen as a $n$-fold compound option, in which the start of a phase depends on the complete success of the previous phase, as illustrated in Fig. 1.

If the initial R\&D investment is successful, then the second phase will be started otherwise, the research will be stopped. Each phase is an option on the next phase. In particular, we focus on Vitosha R\&D project proposed in Cassimon et al. (2011) whose data concerning each phase are summarized in the left side of Table 1. The six-fold compound European option (CEO) without technical risk given in Cassimon et al. (2011) is 264.84 million and the real option value is obtained by subtracting the initial research cost $R_{0}=10.00$ million, i.e. 254.84 million. On the other hand, considering the probability of success $p_{i}$, the six-fold CEO with technical risk is 32.61 million and the real option value becomes 22.61 million.

Due to the computational complexity, Perlitz et al. (1999) and Jensen and Warren (2001) propose to break down the complex R\&D process in two phases, as illustrated in Fig. 2. The simplification of the complex R\&D process with $n$-phases is done to keep mathematics as simple as possible because, in this fashion, the two-period compound option model of Geske (1979) can be used in the absence of technical risk.

However, reducing the drug development process to only two phases implies making a choice where to put the first option exercise time $t_{1}$. For this reason, Cassimon et al. (2011) propose three alternatives in order to transform a six-fold plane into a two-stage 


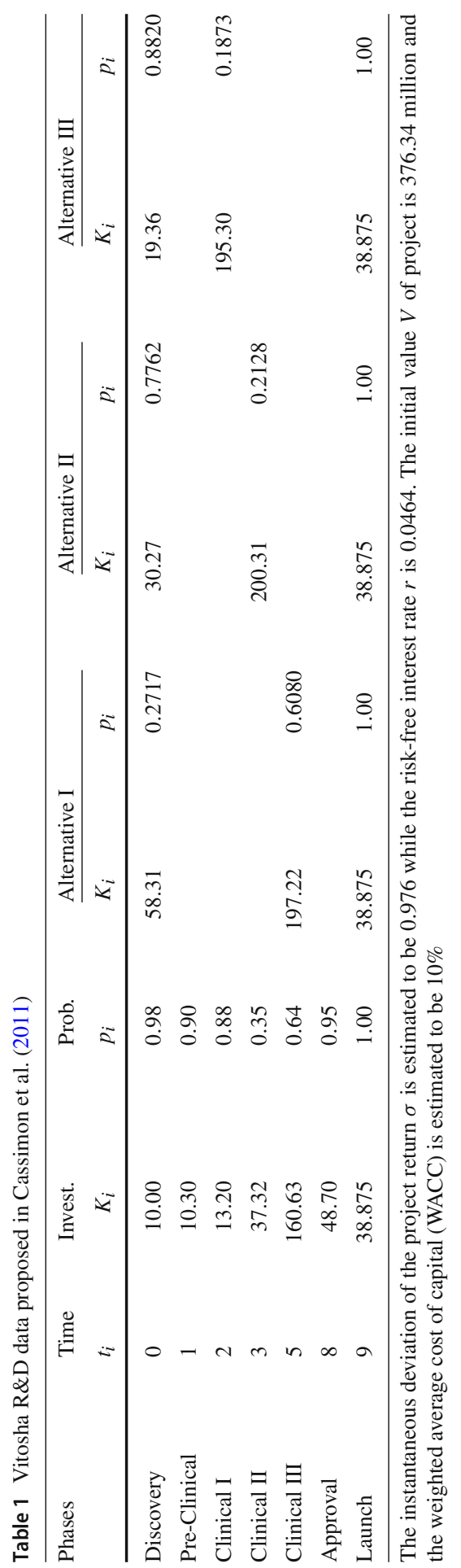




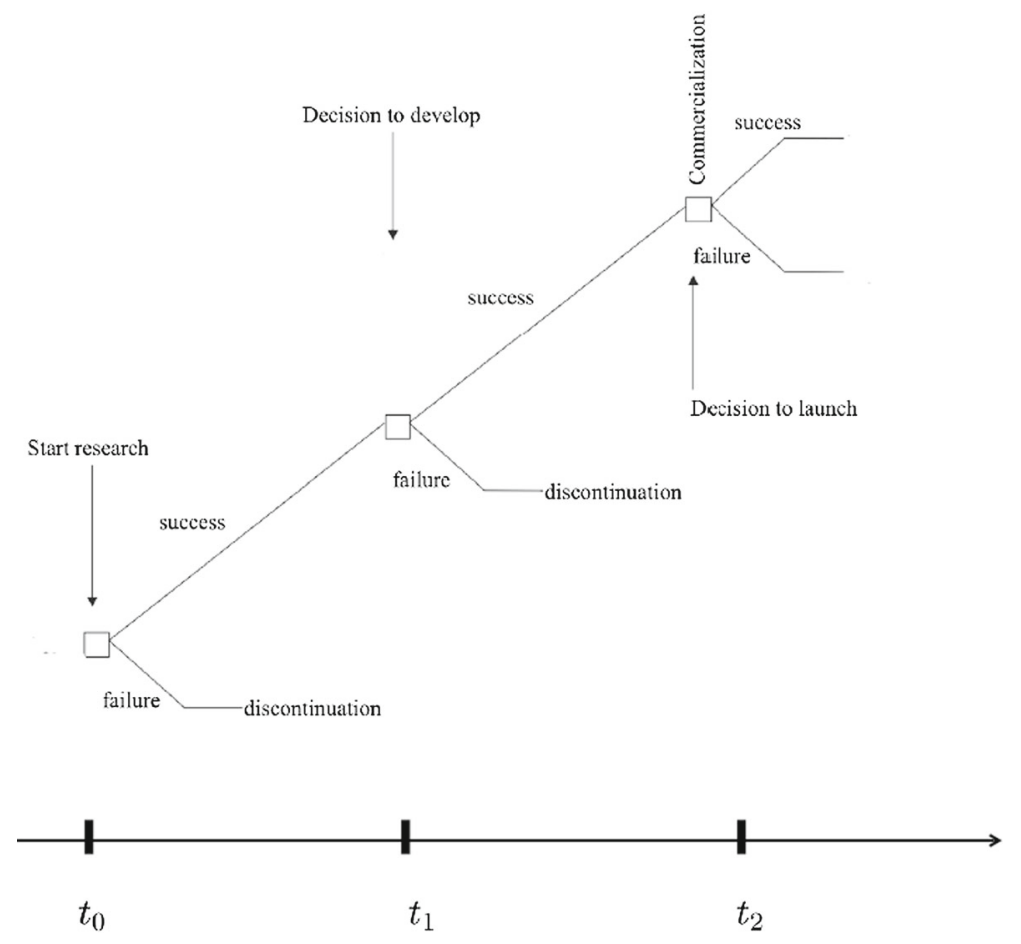

Fig. 2 Structure of an $n=2$ compound option as described in Cassimon et al. (2011)

one. Alternative I considers as the first stage the period between the discovery phase and the drug proof-of-concept (past phase II). In case of success, this stage gives an option on the second phase which is the clinical III phase and the approval phase. Finally, this phase gives an option on the launch of the project by introducing it on the market. So $K_{0}$ is obtained by discounting the costs $K_{i}$ of start of research, pre-clinical test phase, clinical phase I and II using the WACC rate $0.10 ; K_{1}$ is clinical phase III and approval by government and finally $K_{2}$ is the commercialization investment. Entering the first phase (discovery till clinical II) would cost $K_{0}=58.31$ million. Obviously, this cost does not enter into two-fold CEO evaluation but, investing it, management obtains the investment opportunity. The exercise price $K_{1}$ of the first option at time $t_{1}=5$ years is equal to the total cost of finishing the clinical III and the approval phase and is estimated to be $K_{1}=197.22$ million. The exercise price of moving to the second option is the cost of launching the product and it amounts to $K_{2}=38.87$ million, which is the irreversible cost of the follow-up investment in production, marketing, etc. for the market introduction of the product. The time to expiration of the final option is $t_{2}=9$ years; the volatility of the project return is estimated to be $\sigma=0.976$ and the risk-free interest rate amounts 0.0484 . Concerning the technical risk of alternative I, we determine that the success probability of first phase is $p_{0}=0.2717$, namely $0.98 \cdot 0.90 \cdot 0.88 \cdot 0.35$; the probability of success of the second one is $p_{1}=0.6080$, i.e. $0.64 \cdot 0.95$ and finally, the probability of launch is assumed to be equal to $p_{2}=1$. 
Table 2 Real option and two-fold CEO using Geske (1979), Cassimon et al. (2011) and Markov approach

\begin{tabular}{lccc}
\hline & Alternative I & Alternative II & Alternative III \\
\hline Two-fold CEO without risk (Geske) & 307.91 & 273.85 & 250.73 \\
Real option value (Geske) & 249.60 & 243.58 & 231.37 \\
Two-fold CEO with risk (Cassimon) & 50.42 & 37.15 & 28.52 \\
Real option value (Cassimon) & -7.88 & 6.88 & 9.16 \\
Two-fold CEO with risk (Markov) & 80.86 & 70.84 & 61.64 \\
Real option value (Markov) & 22.55 & 40.57 & 42.28 \\
Markov probability $h_{1}$ & 0.6152 & 0.6716 & 0.7224 \\
Markov probability $h_{2}$ & 0.2332 & 0.2417 & 0.2564 \\
Initial probability Markov States 1 and 2 & 0.1358 & 0.3881 & 0.4410 \\
Initial probability Markov States 3, 4 and 5 & 0.2428 & 0.0746 & 0.0393 \\
\hline
\end{tabular}

In the same manner, Cassimon et al. (2011) also propose alternatives II and III about Vitosha project according to Geske (1979) approach whose input data are summarized in the right side of Table 1 .

For this purpose, Table 2 shows, for each alternative, the results of two-fold CEO evaluation taking into account both (Geske 1979) methodology and the (Cassimon et al. 2011) approach. As said previously, the real option value is obtained by subtracting the initial cost $K_{0}$ from the two-fold CEO. Based on real options considerations, in case of technical risk, manager should reject the project in the case of alternative I as it is negative.

Let present our approach in order to value the technical risk using the Markov metholodogy in the case of deterministic decision times. Our approach is based on a different concept of evolution of technical uncertainty and presents a generalization of Cassimon et al. (2011) model. Focusing on the data of altenative I, we analyse a two-fold compound option with $t_{1}=5$ years and $t_{2}=9$ years. We also assume $E=\{1,2,3,4,5\}$ as description of the technical risk of the project and $U_{t_{1}}=\{1,2\}$ and $U_{t_{2}}=\{1\}$.

Using our approach, we have the opportunity to define better the evolution of uncertainty and so to describe in a more efficient manner the evolution of probability. In this way, we propose a more general model that includes several situations. In order to compare the Cassimon et al. (2011) approach, we assume that the success probability of first phase $p_{0}=0.2717$ is split equally between $U_{t_{1}}=\{1,2\}$, i.e. only the states 1 and 2 give the accomplishment of the first phase and, in the same manner, the unsuccess probability $1-p_{0}=0.7283$ is distributed equally among the states 3,4 and 5 . So, a possible distribution of the initial probabilities among the $m=5$ states will be:

$$
\mathbf{p}_{0}^{M}=[0.1358,0.1359,0.2428,0.2428,0.2427]
$$

This means that the first phase is realized with an acceptable success if the Markov chain is in state 1 or 2 being state 1 preferred to state 2 . Consequently, the second phase (market launch) is successful if and only if $X_{t_{2}}=1$. We assume that the generator 
matrix is:

$$
\mathbf{A}=\left(\begin{array}{rrrrr}
-0.50 & 0.40 & 0.10 & 0.00 & 0.00 \\
0.45 & -0.80 & 0.25 & 0.10 & 0.00 \\
0.15 & 0.35 & -0.80 & 0.25 & 0.05 \\
0.05 & 0.35 & 0.35 & -1.00 & 0.25 \\
0.00 & 0.15 & 0.15 & 0.30 & -0.60
\end{array}\right)
$$

Table 2 summarizes the results obtained for three alternatives and the comparison between the two methodologies when the technical risk is considered. For instance, using our approach for the alternative I, we have that the Markov prababilities are $h_{1}=0.6152$ and $h_{2}=0.2332$ and the two-fold CEO with technical risk is 80.86 million and the real option value 22.55 million is obtained subtracting the initial cost $K_{0}=58.31$ million. Obviously, the analysis is carried out for all alternatives.

Some economic results emerge. It is interesting to observe that, when the time to maturity $t_{1}$ was set at the beginning of the clinical II phase and the clinical I phase, as can be expected, reducing the lifetime of the first option reduces the twofold CEO. However, a more complete analysis between the two approaches can be achieved through a sensitivity study considering the values of alternative I. For this purpose, Fig. 3 shows how the two-fold compound option values change when the most important parameters vary. We obseve that, based on the chosen generator matrix $A$, the value of two-fold CEO based on Markov technical uncertainty is greater than the one used by Cassimon et al. (2011). In particular, when the project value $V$, the volatility $\sigma$ and the maturity time $t_{1}$ of realization of investment $K_{1}$ increase, then the values of two-fold CEO option go up. Instead, when the research investment $K_{1}$ and the developing cost $K_{2}$ increase, the values of two-fold CEO decrease.

Finally, Table 3 shows the effects that a different splitting of initial probability distribution $p_{0}$ between the states $\{1,2\}$ produces on the project value. For this reason, we assume $\alpha \in[0,1]$ and the initial distribution of probabilities concerning alternative I is:

$$
\left.\mathbf{p}_{0}^{M}=\left[\alpha p_{0},(1-\alpha) p_{0}, 0.2428,0.2428,0.2427\right)\right]
$$

with $p_{0}=0.2717$. The analysis has been conducted on the three proposed alternatives. The first consideration is that when $\alpha$ increases, i.e. the initial probability of state $E=1$ goes up, the value of the project increases in the three alternatives. Based on our numerical simulations, as State 1 is preferred to State 2, an improving of initial success probability of State 1 increases the two-fold CEO and the real option value. Furthermore, alternative III is more sensitive than alternative II which, in turn, is more sensitive than I. This effect is due to two factors: the first is that the instant $t_{1}$ is closest in alternative III and which therefore increases the technical uncertainty between $t_{1}$ and final time $t_{2}$; the second is that the initial success probability in alternative III is higher than in the other cases. 

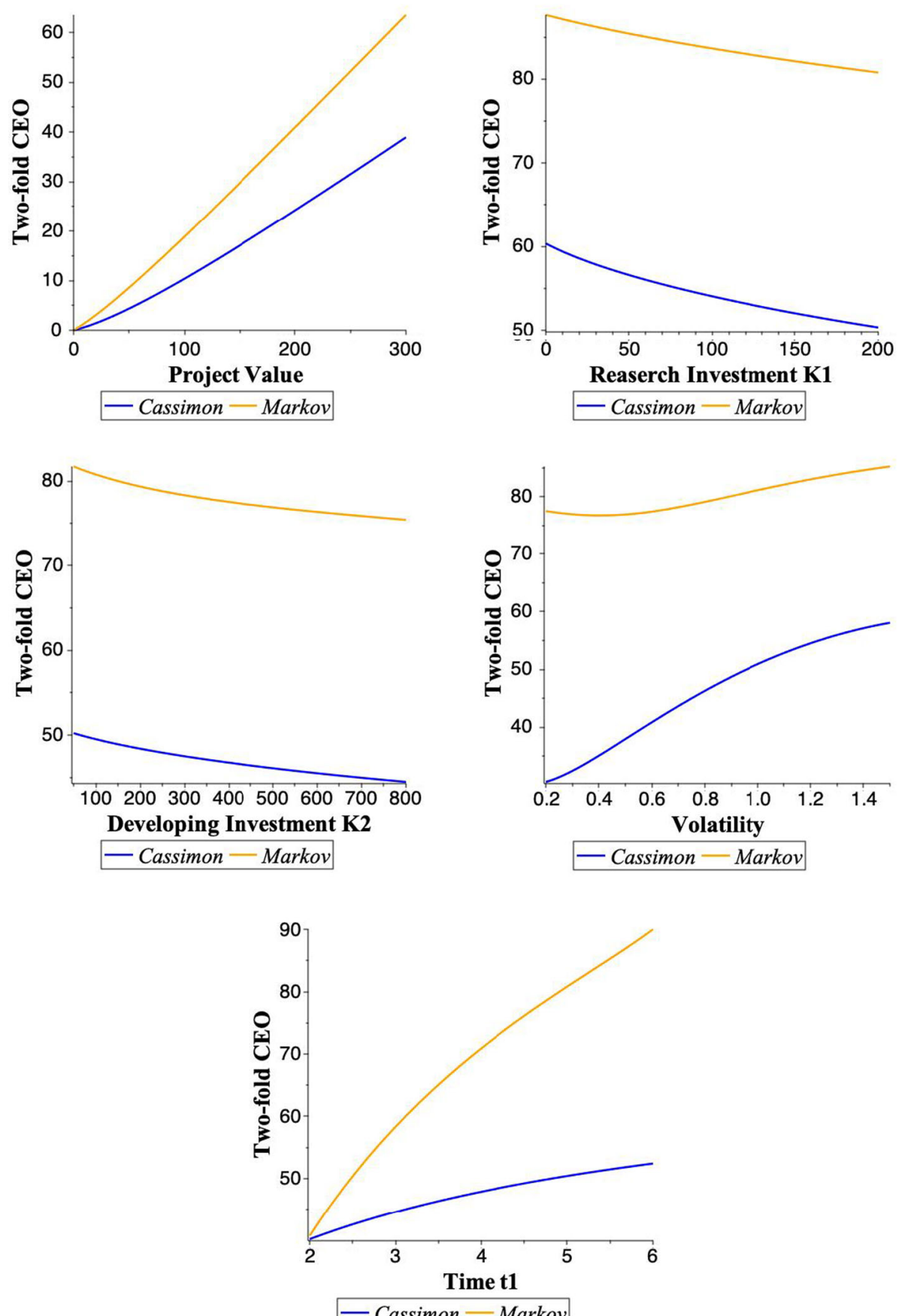

Fig. 3 Sensitivity analysis when the most important parameters change between Cassimon et al. (2011) and Markov approaches using the data of alternative I 


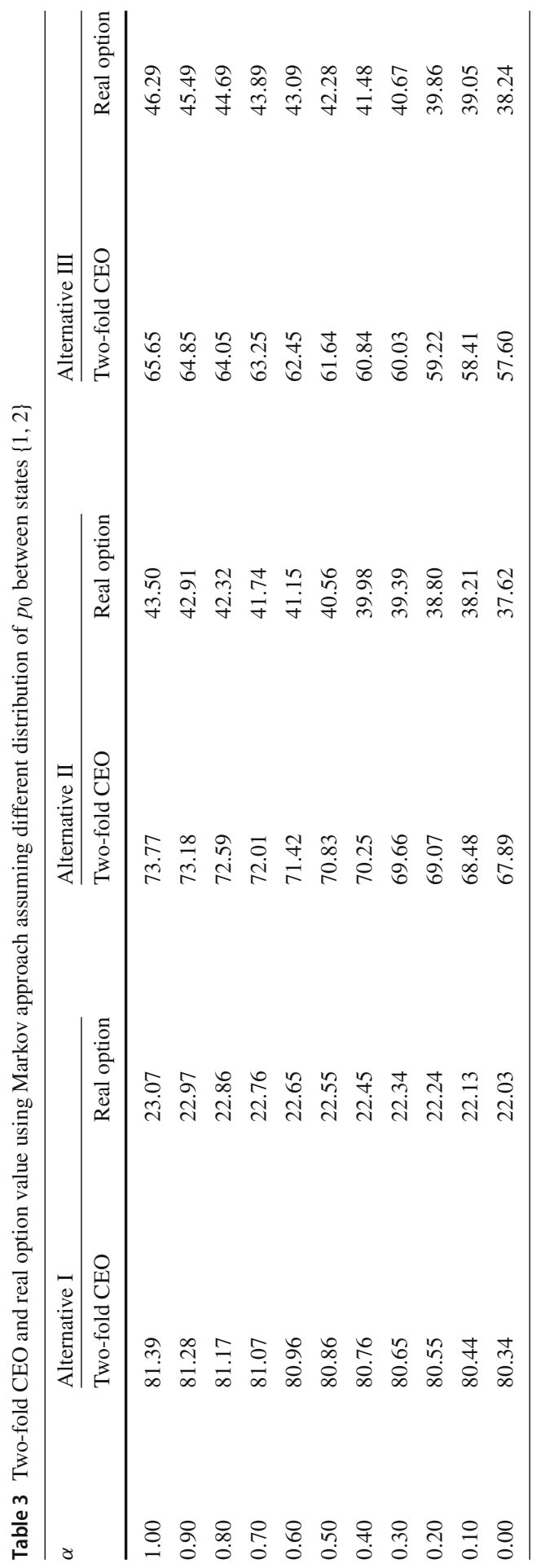




\section{Concluding remarks}

In this paper we have proposed a valuation procedure dealing with depending success probabilities and random developing stage times in order to value multi-phase $\mathrm{R} \& \mathrm{D}$ projects. As we have seen, an $\mathrm{R} \& \mathrm{D}$ project can be considered as an investment made in a phased manner with the commencement of subsequent phase being dependent on the successful completion of the preceding one. For the previous motivation, this kind of investment can be assumed as a Compound Option in which each stage provides information for the next phase creating an opportunity for the subsequent investment. We have argued the importance of technical uncertainty in the valuation of such projects that increases the value of the flexibility that such investments can be postponed, abandoned or increased. Starting from Cassimon et al. (2011), who assume that the success probabilities are independent from each other, we have improved this unrealistic assumption considering that these probabilities are generated according to a a Markov chain and that the decisions times are random variables dependent on the state of the Markov chain. In this fashion, the decision making is more appropriate and complete in order to capture all the managerial flexibility. Finally, we have discussed the Vitosha R\&D pharmaceutical project and we have compared our results with those of Cassimon et al. (2011).

Funding Open access funding provided by Università degli Studi G. D'Annunzio Chieti Pescara within the CRUI-CARE Agreement.

Open Access This article is licensed under a Creative Commons Attribution 4.0 International License, which permits use, sharing, adaptation, distribution and reproduction in any medium or format, as long as you give appropriate credit to the original author(s) and the source, provide a link to the Creative Commons licence, and indicate if changes were made. The images or other third party material in this article are included in the article's Creative Commons licence, unless indicated otherwise in a credit line to the material. If material is not included in the article's Creative Commons licence and your intended use is not permitted by statutory regulation or exceeds the permitted use, you will need to obtain permission directly from the copyright holder. To view a copy of this licence, visit http://creativecommons.org/licenses/by/4.0/.

\section{References}

Amram, M., Kulatilaka, N.: Real Options: Managing Strategic Investment in an Uncertain World. Boston: Harvard Business School Press (1999)

Barbu, V.S., Karagrigoriou, A.: Modeling and inference for multi-state systems. In: Lisnianski, A., Frenkel, I., Karagrigoriou, A. (eds.) Recent Advances in Multi-state Systems Reliability, pp. 59-70. Berlin: Springer (2018)

Borison, A.: Real options analysis: where are the emperor's clothes? J Appl Corporate Finance 17, 17-31 (2005)

Brennan, M.J., Schwartz, E.S.: Evaluating natural resource investments. J Bus 58, 135-157 (1985)

Cassimon, D., De Backer, M., Engelen, P.J., Van Wouwe, M., Yordanov, V.: Incorporating technical risk in compound real option models to value a pharmaceutical R\&D licensing opportunity. Res Policy 40, 1200-1216 (2011)

Cassimon, D., Engelen, P.J., Thomassen, L., Van Wouwe, M.: The valuation of a NDA using a 6-fold compound option. Res Policy 33, 41-51 (2004)

Carr, P.: The valuation of sequential exchange opportunities. J Finance 43, 1235-1256 (1988)

D’Amico, G., Lika, A., Petroni, F.: Change point dynamics for financial data: an indexed Markov chain approach. Ann Finance 15, 247-266 (2019) 
De Blasis, R.: The price leadership share: a new measure of price discovery in financial markets. Ann Finance 16, 381-405 (2020)

Dixit, A.: A general model of R\&D competition and policy. RAND J Econ 19, 317-326 (1988)

Elliott, R.J., Miao, H., Yu, J.: Investment timing under regime switching. Int J Theor Appl Finance 12, 443-463 (2009)

Geske, R.: The valuation of compound options. J Finance Econ 7, 63-81 (1979)

Hartmann, M., Hassan, A.: Application of real option analysis for pharmaceutical R\&D project evaluationempirical results from a survey. Res Policy 35, 343-354 (2006)

Jensen, K., Warren, P.: The use of options theory to value research in the service sector. R\&D Manag 31, 173-180 (2001)

Kellogg, D., Charnes, J.: Real-options valuation for a biotechnology company. Finance Analysts J 56, 76-84 (2000)

Maier, S., Pflug, G.C., Polak, J.W.: Valuing portfolios of interdependent real options under exogenous and endogenous uncertainties. Eur J Oper Res 285, 133-147 (2020)

McDonald, R.L., Siegel, D.R.: Investiment and the valuation of firms when there is an option to shut down. Int Econ Rev 28, 331-349 (1985)

Myers, S.: Finance theory and financial strategy. Interfaces 14, 126-137 (1984)

Newton, D.P., Paxson, D.A., Widdicks, M.: Real R\&D options. Int J Manag Rev 5(6), 113-130 (2004)

Ortobelli Lozza, S., Angelelli, E., Bianchi, A.: Financial applications of bivariate Markov processes. Math Probl Eng (2011)

Perlitz, M., Peske, T., Schrank, R.: Real options valuation: the new frontier in R\&D project evaluation? R\&D Manag 29, 255-270 (1999)

Smith, J., Nau, R.: Valuing risky projects: option pricing theory and decision analysis. Manag Sci 14, 795-816 (1995)

Song, N., Xie, Y., Ching, W., Siu, T.: A real option approach for investment opportunity valuation. J Ind Manag Optim 13, 1213-1235 (2017)

Thomassen, L., Van Castern, J., Van Wouwe, M.: Decomposition of the n-fold compound option. Working Papers 2002040 University of Antwerp (2002)

Trigeorgis, L.: The nature of options interaction and the valuation of investments with multiple real options. J Finance Quant Anal 26, 309-326 (1993)

Weeds, H.: Strategic delay in a real options model of R\&D competition. Rev Econ Stud 69, 729-747 (2002)

Publisher's Note Springer Nature remains neutral with regard to jurisdictional claims in published maps and institutional affiliations. 